\title{
Triathletes Lose Their Advantageous Pain Modulation under Acute Psychosocial Stress
}

\author{
NIRIT GEVA ${ }^{1}$, JENS PRUESSNER ${ }^{2}$, and RUTH DEFRIN ${ }^{1}$ \\ ${ }^{1}$ Department of Physical Therapy and Sagol School of Neuroscience, Sackler Faculty of Medicine, Tel-Aviv University, ISRAEL; \\ and ${ }^{2}$ Douglas Mental Health Institute, Department of Psychiatry, McGill University, Montreal, Quebec, CANADA
}

\begin{abstract}
GEVA, N., J. PRUESSNER, and R. DEFRIN. Triathletes Lose Their Advantageous Pain Modulation under Acute Psychosocial Stress. Med. Sci. Sports Exerc., Vol. 49, No. 2, pp. 333-341, 2017. Introduction: Triathletes, who constantly engage in intensely stressful sport, were recently found to exhibit greater pain tolerance and more efficient pain inhibition capabilities than nonathletes. However, pain inhibition correlated negatively with retrospective reports of mental stress during training and competition. The aim of the current study was to test pain inhibition capabilities of triathletes under acute, controlled psychological stress manipulation. Methods: Participants were 25 triathletes and ironman triathletes who underwent the measurement of pain threshold, pain intolerance, tonic suprathreshold pain, and conditioned pain modulation before and during exposure to the Montreal Imaging Stress Task (MIST). Perceived ratings of stress and anxiety, autonomic variables, and salivary cortisol levels were obtained as indices of stress. Results: The MIST induced a significant stress reaction manifested in the subjective and objective indices. Overall, a significant reduction in pain threshold and in conditioned pain modulation efficacy was observed after the MIST, which reached the baseline levels observed previously in nonathletes. Paradoxically, the magnitude of this stress-induced hyperalgesia (SIH) correlated negatively with the magnitude of the stress response; lowstress responders exhibited greater SIH than high-stress responders. Conclusion: The results suggest that under acute psychological stress, triathletes not only react with $\mathrm{SIH}$ and a reduction in pain modulation but also lose their advantageous pain modulation over nonathletes. The stronger the stress response recorded, the weaker the SIH. It appears that triathletes are not resilient to stress, responding with an increase in the sensitivity to pain as well as a decrease in pain inhibition. The possible effects of athletes' baseline pain profile and stress reactivity on SIH are discussed. Key Words: ATHLETES, PAIN PERCEPTION, PAIN MODULATION, CORTISOL, STRESS REACTIVITY
\end{abstract}

$\mathrm{T}$ riathletes and ironman triathletes engage in extremely intense physical exercise such as sequential and continuous swimming, cycling, and running for miles (4). Both training and competitions involve a considerable amount of physical and psychological stress and pain over several hours daily $(4,14)$. Whereas the acute effect of physical exercise on the pain system of athletes has been studied in depth (18), the effect of acute psychological stress has hardly been studied. Stress is currently defined as a cognitive perception of uncontrollability and/or unpredictability that is expressed in physiological and behavioral responses (19). Triathletes report of high levels of psychological stress during training and competitions, ranging from 4 to 7 on a $0-10$ scale (14). This group of athletes thus provides a unique opportunity to

Address for correspondence: Ruth Defrin, Department of Physical Therapy, Sackler Faculty of Medicine, Tel-Aviv University, Tel-Aviv 69978, Israel; E-mail: rutidef@post.tau.ac.il. study the interactions between intense exercise, stress, and the pain system.

In a previous study assessing the baseline pain perception of triathletes, we found that they exhibited greater pain tolerance, lower pain ratings, and more efficient pain inhibition capabilities compared with nonathlete controls (14). This sensory profile of an increased ability to modulate pain is probably related to triathletes' exceptional performances. However, we also found that the magnitude of pain inhibition inversely correlated with the levels of reported psychological stress during training and competitions so that the higher the reported stress, the less efficient was pain inhibition (14). Notably, the stress levels in the aforementioned study were recorded retrospectively using self-report scales. There is thus a need to validate these reports and to verify causal relationship between stress and pain modulation in this population. We were unable to find other studies in which the effect of acute psychological stress on pain perception was evaluated among athletes.

The effect of acute psychological stress on pain perception of nonathletes on the other hand has been widely studied yielding inconsistent results. Several studies reported an increase in pain threshold/tolerance after psychological stress, a phenomenon termed "stress-induced analgesia" (SIA) $(1,12)$, whereas others reported enhancement in pain, i.e., stress-induced hyperalgesia 
(SIH) $(7,9)$. Fewer studies tested additional aspects of the pain system, showing a reduction (26) or no effect of stress on pain inhibition (8) or on pain excitation $(8,9)$. Using a validated psychological stress manipulation, we recently reported that acute stress reduced the pain inhibition and increased pain excitation among nonathletes (15). Furthermore, the effect correlated positively with the degree of perceived stress induced by the manipulation; the higher the acute stress response, the stronger was the effect. The question arises whether triathletes, who have more efficient pain modulation capabilities than nonathletes at baseline, respond to stress in a similar manner or whether their pain modulation capacity is "resilient" to acute psychological stress manipulation. The aim of the present study was therefore to investigate the manner by which pain perception and pain modulation of triathletes are affected by acute psychological stress.

\section{METHODS}

\section{Subjects}

Participants were 25 male triathletes and ironman triathletes (mean \pm SD age $=35.9 \pm 10 \mathrm{yr}$ ). We included only male subjects to avoid the confounding effect of sex on both pain modulation and stress perception and regulation. The subjects were recruited by advertisements posted at the university and Internet sites of triathlon. Inclusion criteria were as follows: a training routine of at least $7 \mathrm{~h} \cdot \mathrm{wk}^{-1}$ and participation in at least five triathlon and/or ironman triathlon competitions per year. Exclusion criteria were acute or chronic pain, present or previous pathology in the hands (testing site), bruises or any other skin lesions on the hands, diseases causing potential neural damage (e.g., diabetes), and systemic and mental illnesses (e.g., anxiety disorders, depression, and bipolar disorder). Informed consent was obtained from all the participants after explaining the goals of the study. The experiment was approved by the Helsinki committee of Sheba Medical Center and the institutional review board of Tel-Aviv University.

\section{Equipment}

Thermal stimulators. Heat stimuli were delivered using two Peltier-based computerized thermal stimulators (TSA II; Medoc Ltd., Israel), with $3 \times 3 \mathrm{~cm}$ contact probes. According to the principles of the Peltier element, a passage of current through the Peltier element produces temperature changes at rates determined by an active feedback system. As soon as the target temperature or designated sensation is attained, probe temperature actively reverts to a preset adaptation temperature by passage of an inverse current. The adaptation (baseline) temperature was set to $35^{\circ} \mathrm{C}$, and the maximal temperature was $52^{\circ} \mathrm{C}$. The probes were attached to the testing site using a Velcro band.

Recording and processing of physiological signals. The physiological signals were recorded, sampled, and stored using a personal computer with the PMD-100 system (Medasense Biometrics Ltd., Ramat Yishay, Israel). A one-lead ECG signal was sampled with a frequency of $500 \mathrm{~Hz}$, and a reflectance-mode Photo-plethysmograph (PPG) signal from the right-hand index finger was sampled with the same frequency. Skin conductance (measured in microsiemens) was measured using two electrodes positioned on the volar pads of the distal phalanx in index finger and was sampled with a frequency of $31.25 \mathrm{~Hz}$. The recorded signals were synchronized and processed offline using Matlab R2010 scientific software (The Mathworks, Inc., Natick, MA).

\section{The Montreal Imaging Stress Task}

The Montreal Imaging Stress Task (MIST) was used to induce acute psychological stress. The MIST is a reliable and versatile tool for inducing stress perception and physiological responses (10). The principal component of the MIST is a computer program that displays a mental arithmetic task, a rotary dial for submission of a response, a text field that provides feedback on the submitted response ("correct," "incorrect," or "timeout"), and two default performance indicators, one for the individual subject's performance and one for average performance of all subjects. The program runs for 8 min during which the tasks appear one by one. Each task is time limited; the elapsed time is displayed by a progress bar moving from left to right on the computer screen, with the exact time allowed for each task depending on the subject's previous performance. The program continuously records the subject's average response time, and the number of correct responses and adjusts the time limit continuously to enforce a range of approximately $20 \%$ to $45 \%$ correct answers. Before the task, the investigator informs the subject that the average performance is approximately $80 \%-90 \%$ correct answers and that his individual performance should be close or equal to the average performance of all subjects if the subject's data are to be used in the study. Finally, the subject is told that the investigator is after his performance but cannot help or talk with him and that the director of research is after the performance on a second monitor in the control room. After the end of the session, the investigator informs the subject about a poor performance and asks him to try again to do his best. After completion of the second session, the subject receives again a negative feedback about his performance.

\section{Indices of the Stress Response}

Perceived stress. Perceived stress was evaluated using a visual analog scale (VAS). The VAS consisted of a $10-\mathrm{cm}$ line with two anchor points at its extremes, set as $0=$ no stress and $10=$ most intense stress imaginable.

State anxiety. Anxiety was evaluated with the short form of the State-Trait Anxiety Inventory (32). This questionnaire contains 10 items, and subjects are asked to rate the degree to which they experienced each symptom of anxiety at that moment on a four-point Likert-type scale ( 1 = not at all to $4=$ very much so). This measure of state anxiety has 
been used extensively in previous research and has consistently demonstrated good psychometric properties, especially under conditions of stress.

Autonomic variables. The sympathetic-adrenal-medullary system responds to stress by secreting noradrenaline thereby increasing sympathetic tone, resulting in, e.g., an increase in HR and skin conductance and a decrease in heart rate variability (HRV). The sympathetic response was thus investigated by recording the change in $\mathrm{HR}, \mathrm{HRV}$, and galvanic skin response (GSR) using the PMD-100 system. HR, HRV, and GSR were recorded continuously during the experiment, and the values were extracted offline for relevant time points as described in the following sections.

Salivary cortisol. The hypothalamic-pituitary-adrenal axis is strongly activated by psychosocial stress and secretes the stress hormone cortisol (11). Saliva samples of cortisol were collected with Salivettes (Sarstedt, Rommels-dorft, Germany). Participants were asked to place a Salivette (cotton roll) in their mouths, chew on it for a minute until it became saturated, and place it in storage container. The samples were then stored at $-20^{\circ} \mathrm{C}$ up to 3 months, until assayed. Cortisol levels were assayed using a commercial ELISA kit (Assay Design, Ann Arbor, MI). Measurements were performed in duplicate, according to the kit's instructions with the reagents provided. Cortisol levels were calculated using MatLab 7, according to standard parametric calibration curves based on the data from the kit (20).

\section{Quantitative Sensory Testing of Pain Perception and Modulation}

Pain threshold and pain tolerance. Pain threshold and pain tolerance were measured with the method of limits, using the thermal stimulator. For each threshold measurement separately, subjects received four successive ramps of gradually increasing temperature, starting from a baseline temperature of $35^{\circ} \mathrm{C}$, at a rate of $2{ }^{\circ} \mathrm{C} \cdot \mathrm{s}^{-1}$ (interstimulus interval of $30 \mathrm{~s}$ ). For pain threshold, the subjects were asked to press a switch when a pain sensation was first perceived. For pain tolerance, the subjects were asked to press a switch when they could no longer withstand the pain. Pressing the switch resulted in an automatic recording of the threshold temperature and reset the probe temperature to baseline value. Pain threshold and pain tolerance were computed separately by averaging the readings of four successive stimuli in each measurement (15).

Perceived pain intensity. Perceived pain intensity was measured with the method of direct scaling, using the thermal stimulator and a VAS. The VAS end points were set as $0=$ "no pain sensation" and $10=$ "the most intense pain sensation imaginable." Subjects received a series of thermal stimuli and were asked to rate their pain after each stimulus on the VAS. The stimulus intensities, presented in an ascending manner in steps of $1^{\circ} \mathrm{C}$, rose from a baseline temperature of $35^{\circ} \mathrm{C}$ (rate of rise $2^{\circ} \mathrm{C} \cdot \mathrm{s}^{-1}$ ), to a destination temperature ranging from $37^{\circ} \mathrm{C}$ to the intensity eliciting 6 on the VAS, at which it remained for $1 \mathrm{~s}$ and then returned to baseline. An interstimulus interval of $45 \mathrm{~s}$ was maintained to avoid any changes in skin sensitivity and to allow for adequate VAS scoring. Individual stimulus-response functions were obtained for each subject, and the temperature eliciting a value of 5 on the VAS was extracted from the functions to be used in the subsequent testing described in the following section (14).

Conditioned pain modulation index of pain inhibition. Conditioned pain modulation (CPM) is an experimental paradigm that is designed to evaluate the efficacy of a descending pain inhibition pathway termed "diffuse noxious inhibitory control (DNIC)." The DNIC pathway, mediated by the brain stem subnucleus reticularis dorsalis, causes pain reduction in a particular body region by noxious stimuli applied in an another remote body region $(39,40)$. CPM was measured by applying a noxious stimulus to one forearm (the "test stimulus" [TS]) and evaluating its perceived intensity alone, and in the presence of another noxious stimulus applied to the contralateral forearm (the "conditioning stimulus" [CS]). The two stimuli were administered with two thermal stimulators. The TS was noxious heat at an intensity equivalent to 5 on the VAS (individually adjusted), applied to the volar aspect of the forearm for duration of $10 \mathrm{~s}$. The CS was noxious heat at an intensity equivalent to 5 on the VAS, applied to the volar aspect of the contralateral forearm for $25 \mathrm{~s}$. The second application of the TS occurred $15 \mathrm{~s}$ after the application of the CS. The subjects were asked to rate the TS immediately upon its termination. The magnitude of CPM was calculated by subtracting the VAS rating of the TS in the presence of the CS from the VAS rating of the TS alone (15).

Tonic suprathreshold pain index of pain enhancement. The complementary test of CPM is tonic suprathreshold pain (TSP), an experimental paradigm that is designed to evaluate the excitability of the pain system, specifically the function of spinal nociceptive neurons that respond to repeated/constant noxious stimuli of fixed intensity by a gradual increase in their output, the "windup" phenomenon (33). TSP was measured by administering to the forearm a noxious heat stimulus at temperature equivalent to 5 on the VAS for $20 \mathrm{~s}$. Subjects were asked to rate the amount of perceived pain (using VAS) at the beginning of the stimulus and after $15 \mathrm{~s}$. The magnitude of TSP was calculated by subtracting the first VAS rating from the last (14).

\section{Procedure}

Each subject was invited to a single testing session that lasted approximately $3 \mathrm{~h}$. The subjects were instructed to avoid physical exercise $24 \mathrm{~h}$ before the testing day as well as on the testing day itself and to refrain from food and caffeine $1 \mathrm{~h}$ before testing. Because cortisol levels normally fluctuate throughout the day, all subjects were tested beginning at 1 PM. Testing took place in a quiet room. Temperature in the room was maintained at $22^{\circ} \mathrm{C}$. The subject sat in a comfortable armchair. After signing an informed consent, the subject was trained in the psychophysical and endocrine measurements. 
After a short break, the subject was connected to the monitor sensors that remained active thereafter for the entire experiment.

Figure 1 describes the experimental protocol. Similarly to our previous study (15), there were four main epochs from which data were obtained, as follows:

A) Baseline. The subject was asked to rest quietly for $10 \mathrm{~min}$ (from which time baseline autonomic variables were extracted) immediately after which the first saliva sample was taken, and the first perceived stress and the state anxiety scores were obtained.

B) Prestress pain measurements. The subject underwent a series of testing that included the measurement of pain threshold, pain tolerance, perceived pain intensity, CPM, and TSP. Upon completion of these measurements, the second saliva sample was taken, and the second perceived stress and the state anxiety scores were obtained to evaluate whether pain testing induced a stress effect.

C) During stress (MIST) pain measurements. The subjects were explained how to operate the software of the MIST and received the preparatory explanations for the stress manipulation after which the software was run for $8 \mathrm{~min}$. At the end of the task, the subjects received a negative feedback about their performance and were asked to perform the task again for additional $8 \mathrm{~min}$ after which they received again a negative feedback about their performance. Immediately upon completion of the two MIST tasks, the third saliva sample was taken, and the third perceived stress and the state anxiety scores were obtained to evaluate whether the manipulation induced stress. Afterward, the same sensory testing as in epoch B was performed in the same order. It was important to maintain the order of measurements before and after the stress manipulation to avoid additional influencing factors.

D) Recovery. Upon completion of the pain measurements, the subjects received an explanation of the true purpose of the MIST and were assured that their performed was satisfactory. They were informed that the experiment is over and were asked to relax and rest. Approximately 20 min after the reassurance, the forth saliva sample was taken and the forth perceived stress, and state anxiety scores were obtained to evaluate whether stress response had subsided.

\section{Data Analysis}

Data were analyzed using IBM SPSS statistic software version 21. The sample size was estimated a priori based on our previous experience with the psychophysical testing and on the expected difference between baseline and stress condition in the mean values of the main outcome measures: pain threshold and CPM. For $\alpha=0.05$ and statistical power of $80 \%$, the calculation yielded a sample size of 18 subjects and for $\alpha=0.01,24$ subjects. Continuous variables were described as means \pm SD and categorical variables as counts and $\%$. All data underwent the Kolmogorov-Smirnov analysis for normality of distribution. Parametric and nonparametric analyses of variance with corrected post hoc tests were used to evaluate the effect of condition (baseline, prestress, stress, and recovery) on perceived stress, perceived anxiety, HR, HRV, GSR, cortisol, and the sensory tests (pain threshold, pain tolerance, CPM, and TSP). The magnitude of change in the sensory indices after the stress manipulation was calculated by subtracting the level measured during stress from that measured at baseline. The correlations between changes in the sensory indices (delta values) and between the stress and the demographic indices were calculated using Pearson's correlation coefficients. $P<0.05$ was considered significant.

\section{RESULTS}

\section{Characteristics of the Study Groups}

Triathletes reported that they had engaged in triathlon and/or ironman triathlon sport for the last $8.6 \pm 6.9 \mathrm{yr}$ (range $1-24 \mathrm{yr}$ ), had participated in $12.32 \pm 7.2$ competitions on average (range 5-42) every year, and had trained for $16.43 \pm$ $9.8 \mathrm{~h} \cdot \mathrm{wk}^{-1}$ (range $7-31.5 \mathrm{~h}$ ). The triathletes reported that they experience moderate to high levels of physical and mental stress during training $(5.04 \pm 2.8$ and $4.25 \pm 3.2$ VAS units, respectively) and competition $(6.5 \pm 3.5$ and $5.7 \pm 2.7$ VAS units, respectively).

\section{Validation of the Stress Response}

Table 1 presents the values of the stress indices in the four conditions of the study: rest, prestress pain measurement, stress (post-MIST), and recovery, as well as comparisons between the conditions ( $t$-tests). A significant main effect of condition

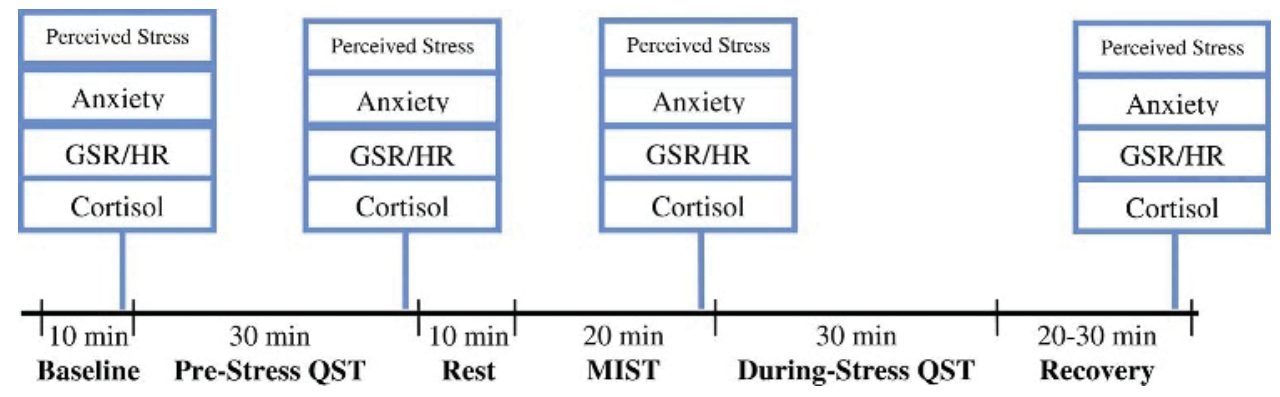

FIGURE 1-The experimental protocol. QST, quantitative sensory testing. 
TABLE 1. Stress indices obtained in the four epochs of the study.

\begin{tabular}{|c|c|c|c|c|c|c|}
\hline & Baseline & Prestress Measurements & Stress & Recovery & $F$ & $P$ \\
\hline Stress $(0-10)$ & $1.2 \pm 1.4^{\star, a}$ & $0.82 \pm 1^{* \star * *, b}$ & $6.89 \pm 2.9^{\star \star \star \star, C}$ & $0.94 \pm 1.6^{\star \star \star \star, d}$ & 98.32 & $<0.000$ \\
\hline State anxiety (10-40) & $12.88 \pm 2.9^{\star, a}$ & $11.96 \pm 2^{\star \star \star \star, b}$ & $22.36 \pm 6.9^{\star \star \star \star, c}$ & $13 \pm 2.9^{\star \star \star \star, d}$ & 78.74 & $<0.000$ \\
\hline $\mathrm{HR}$ (bpm) & $54.86 \pm 6.1^{\star *, a}$ & $57.11 \pm 6.6^{*, b}$ & $64.75 \pm 26.7^{\star \star, C}$ & $51.19 \pm 14.1^{\star \star \star \star, d}$ & 2.18 & 0.1 \\
\hline GSR (mmol) & $5.05 \pm 4^{\star \star \star *, a}$ & $11.2 \pm 7.7^{\star \star \star \star, b}$ & $12.94 \pm 10.1^{\star \star \star \star, C}$ & $10.23 \pm 8.2^{\star \star, d}$ & 15.07 & $<0.000$ \\
\hline Cortisol $\left(\mathrm{pg} \cdot \mathrm{mL}^{-1}\right)$ & $670.3 \pm 399$ & $751.66 \pm 769^{*, b}$ & $1085.88 \pm 969^{*, C}$ & $645.4 \pm 491^{\star \star, d}$ & 2.35 & 0.085 \\
\hline
\end{tabular}

Values are presented as mean $\pm \mathrm{SD}, F$ values, and $P$ values from the ANOVA.

aPaired comparisons between baseline and prestress measurements.

${ }^{b}$ Paired comparisons between prestress and during stress measurements.

${ }^{c}$ Paired comparisons between baseline and during stress measurements.

${ }^{d}$ Paired comparisons between during stress and recovery measurements.

${ }^{*} P<0.05$.

${ }^{* *} P<0.01$.

$\star \star * P<0.001$

$\star * * * P<0.0001$

was found on perceived stress, perceived anxiety, and GSR, and a borderline effect was also found on cortisol levels. Post hoc tests revealed that the level of perceived stress, perceived anxiety, HR, GSR, and cortisol significantly increased during stress as compared with baseline and prestress condition and then decreased to baseline values in recovery. The level of HRV decreased during stress as compared with baseline and prestress condition and then increased back to normal in recovery.

\section{The Effect of Stress on Pain Perception}

Pain threshold and pain tolerance. A significant effect of stress was found on pain threshold, which decreased compared with baseline $(P>0.01)$ (Fig. 2A). By contrast, stress did not affect pain tolerance threshold, which remained steady during baseline and stress conditions, respectively (Fig. 2B). The reduction in pain threshold correlated with the increase in perceived stress $(r=-0.48, P<0.01)$ and perceived anxiety $(r=-0.53, P<0.01)$. Namely, the greater the stress response of the individuals, the smaller was the SIH.

CPM. A significant main effect of condition $\left(F_{1,47}=\right.$ 27.04, $P<0.0001$ ) was found on the pain ratings of the TS; CPM magnitude reduced significantly during stress (delta of $1.72 \pm 2$ VAS units) as compared with baseline condition (delta of $3.15 \pm 1.7, P<0.0001$ ) (Fig. 2 C). The reduction in CPM due to stress correlated negatively with HRV during stress $(r=-0.48, P<0.05)$ and with the increase in perceived stress due to the stress manipulation $(r=-0.39$, $P<0.05)$. Namely, the greater the stress response of the individuals, the smaller was the reduction in CPM.

TSP. Stress did not affect TSP $\left(F_{2,46}=0.19, P=0.90\right)$. At baseline, pain ratings increased from $4.38 \pm 1.5$ to $5.04 \pm$ 2.3 VAS units $(P=0.1)$ and during stress, from $4.43 \pm 1.3$ to $5.03 \pm 2.4$ VAS units $(P=0.08)$, namely, a similar magnitude of $0.67 \pm 2.5$ and $0.6 \pm 2$ VAS units, respectively (not shown).

\section{High- and Low-Stress Responders}

Because of the correlations between the change in perceived stress after the manipulation and between pain threshold/CPM, and to further explore the relationship between the reactivity to stress, pain perception, and baseline characteristics of the triathletes, we divided the group into two subgroups according to their perceived stress level. We chose perceived stress for this purpose and not the other, objective indices of stress because this variable represents the current definition of stress (19). Accordingly, low-stress responders $(n=9)$ had below group mean perceived stress level $(4.38 \pm 2.0$ VAS units $)$, and high-stress responders $(n=16)$
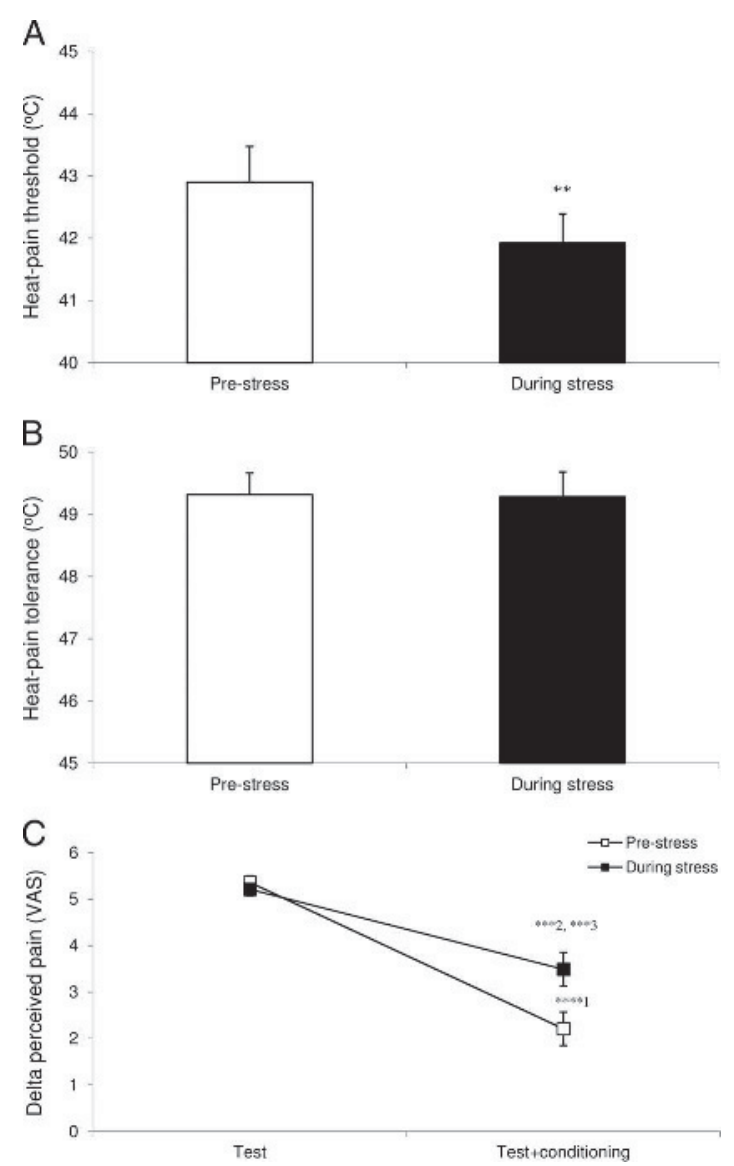

FIGURE 2-Pain perception and modulation tests pre- and poststress manipulation. A, Pain threshold significantly decreased after the stress manipulation as compared with baseline $(* * P<0.01)$. B, Pain tolerance was not affected by the stress manipulation. $\mathrm{C}, \mathrm{A}$ significant $\mathrm{CPM}$ was induced in both prestress and during stress condition $\left({ }^{1} * * * * P<0.0001\right.$ and ${ }^{2} * * P<0.001$, respectively); however, the magnitude of CPM significantly decreased after the stress manipulation as compared with prestress condition $\left({ }^{3 * * * P}<\mathbf{0 . 0 0 1}\right)$. Values denote mean \pm SEM. 


\begin{tabular}{|c|c|c|c|}
\hline & Low-Stress Responders & High-Stress Responders & $P$ \\
\hline Perceived mental stress during training* & $3.11 \pm 3.9$ & $4.93 \pm 2.6$ & $<0.05$ \\
\hline Perceived physical stress during training* & $3.67 \pm 3.6$ & $5.87 \pm 1.8$ & $<0.05$ \\
\hline Perceived mental stress during competitions* & $3.75 \pm 2.7$ & $6.87 \pm 1.9$ & $<0.001$ \\
\hline Perceived stress at baseline (experiment day) ${ }^{*}$ & $0.49 \pm 0.6$ & $1.59 \pm 1.6$ & $<0.05$ \\
\hline Perceived anxiety at baseline (experiment day) & $11.1 \pm 1.4$ & $13.88 \pm 3.1$ & $<0.01$ \\
\hline Cortisol at baseline (experiment day) $\left(\mathrm{pg} \cdot \mathrm{mL}^{-1}\right)$ & $854.96 \pm 691$ & $593.35 \pm 191$ & 0.11 \\
\hline Delta perceived stress (baseline vs stress) ${ }^{\star}$ & $3.89 \pm 2.1$ & $6.78 \pm 1.7$ & $<0.001$ \\
\hline
\end{tabular}

Values are presented as mean $\pm \mathrm{SD}$.

*Scale 0-10.

had above group mean perceived stress level $(8.37 \pm 0.6$ VAS units) $(P<0.0001)$. Low- and high-stress responders did not differ in age or in training/competition variables.

Table 2 describes the characteristics of the low- and highstress responders with regard to their perceptions toward stress during triathlon training and competitions and in the experiment day, at baseline and after the stress manipulation. The low-stress responders reported lower stress levels in everyday life activities related to the triathlon (training and competitions) and also lower stress levels in the experiment compared with high-stress responders. In addition, the changes in perceived stress (Fig. 3A) and in cortisol level (Fig. 3B) after the stress manipulation was significantly smaller in the low-stress compared with the high-stress responders. In fact, the former did not exhibit any significant increase in cortisol after the manipulation. Note that low-stress responders also had a lower level of perceived stress at baseline compared with high-stress responders (Fig. 3A).

With reference to the psychophysical testing, the pain threshold decreased significantly after stress manipulation among both low- and high-stress responders to a similar degree (delta of $0.83^{\circ} \mathrm{C} \pm 0.8^{\circ} \mathrm{C}, P<0.01$, and $1.05^{\circ} \mathrm{C} \pm 2.5^{\circ} \mathrm{C}$, $P<0.05$, respectively) (not shown). Pain tolerance, on the other hand, decreased only among low-stress responders (delta of $\left.-0.47^{\circ} \mathrm{C} \pm 0.5^{\circ} \mathrm{C}, P<0.05\right)$ and did not change among the high-stress responders (delta of $+0.21^{\circ} \mathrm{C} \pm 0.8^{\circ} \mathrm{C}, P=0.1$ ) (Fig. 4A). The difference in the change in pain tolerance between low- and high-stress responders was significant $(P<0.05)$.

CPM decreased significantly in both low- and high-stress responders because of stress conditions (delta of $-2.39 \pm 2$ and $-0.89 \pm 1$ VAS units, between baseline and stress condition $P<0.05$, respectively), but the reduction in CPM in low-stress responders was significantly more pronounced $(P<0.05)$ (Fig. 4B). It is noteworthy that low-stress responders had significantly higher CPM at baseline compared with high-stress responders $(P<0.001)$ (Fig. 4B). Nevertheless, the percent change in CPM (between baseline and stress condition) in low- and high-stress responders was $-60.15 \%$ and $-34.28 \%$, demonstrating an almost twofold decline in CPM in low- versus high-stress responders.

TSP was not significantly affected by the stress manipulation in either low- or high-stress responders. Interestingly, only high-stress responders exhibited TSP both at baseline $(+1.46 \pm 2.2$ VAS units) and during stress $(+1.14 \pm 2.2)$, whereas low-stress responders did not present any change in pain ratings in the TSP test $-0.39 \pm 1.6$ and $-0.06 \pm 2.4$ VAS units, respectively) (not shown).

\section{DISCUSSION}

The results suggest that SIH manifested in the reduction in pain threshold and reduction in CPM. Interestingly, however, SIH correlated negatively with the magnitude of the subjects' stress response. This was observed at a group level, in which SIH was smaller as stress response was stronger, and also after the division into two subgroups wherein greater SIH was noted among low- versus highstress responders. This seemingly paradoxical profile suggests that the response of triathletes to psychological stress is complex, as discussed in the following section.

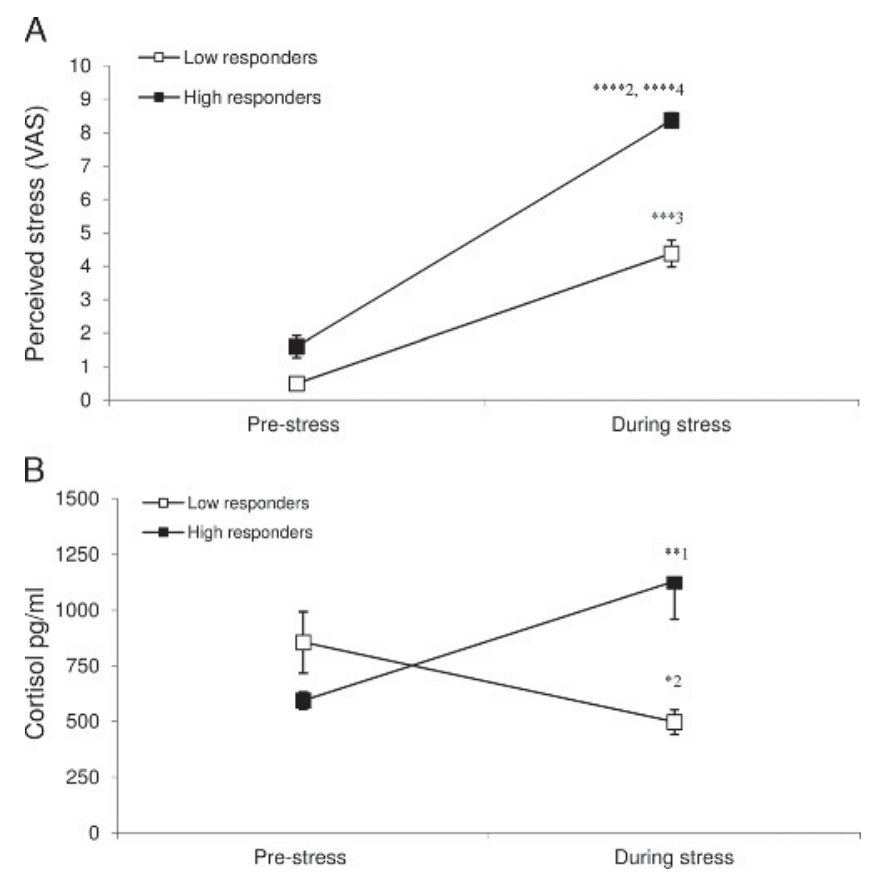

FIGURE 3-Stress indices among low- and high-stress responders. A, Baseline perceived stress was significantly higher among high- versus low-stress responders $\left({ }^{1} * P<0.05\right)$. Both subgroups exhibited an increase in perceived stress after the manipulation $(2 * * * * P<0.0001$ and $3 * * * P<0.001$, respectively); however, this increase was larger among high-stress responders $\left({ }^{4} * * * * P<0.0001\right)$. B, Cortisol level of high-stress responders significantly increased after the stress manipulation $\left({ }^{1 * *} \boldsymbol{P}<\right.$ 0.01), whereas that of low-stress responders did not change, leading to a significant subgroup difference in cortisol after the manipulation $\left({ }^{2} * \boldsymbol{P}<\right.$ 0.05). Values denote mean \pm SEM. 
A

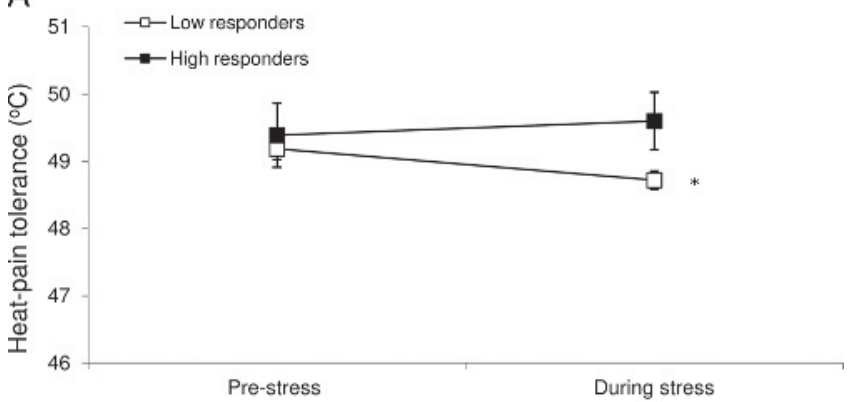

B

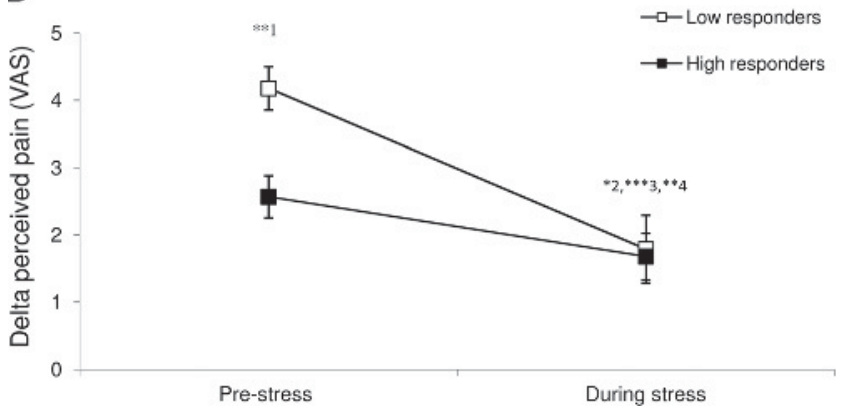

FIGURE 4-Pain perception and modulation tests pre- and poststress manipulation among low- and high-stress responders. A, Heat pain tolerance that was similar in high- and low-stress responders at baseline decreased significantly after the stress manipulation only among lowstress responders $(* P<0.05) . \mathrm{B}, \mathrm{CPM}$ that was significantly better in low-stress responders at baseline $\left({ }^{1 * * P}<0.01\right)$ decreased among both high-stress $(2 * P<0.05)$ and low-stress $\left({ }^{3} * * P<0.01\right)$ responders; however, the reduction was significantly greater among the latter group $\left({ }^{4} * * P<0.01\right)(B)$. Values denote mean \pm SEM.

Stress-induced hyperalgesia. In our previous study (14), the CPM was more efficient in triathletes than that in the controls, a trait that may underlie their ability to persevere in intense physical efforts despite the involved pain. Yet the previous study also demonstrated that the level of CPM among triathletes inversely correlated with the level of their perceived psychological stress during training and competitions; the higher the reported stress, the less efficient was CPM. Because psychological stress in the previous study was based on retrospective self-reports, the purpose of the present study was to induce an acute, controlled psychological stress and to test its effect on pain perception and pain modulation. We were interested to learn whether the very efficient baseline pain modulation of triathletes will render them more resilient to stress. The results show that triathletes are by no means resilient to stress; rather, they respond with an increase in the sensitivity to pain as well as a decrease in pain inhibition.

The SIH seen here among athletes correspond with SIH seen among nonathletes after the same (15) or other stress manipulations $(7,9,26)$. However, several studies have also reported the opposite phenomenon to SIH, namely, SIA that manifested in the elevation of pain threshold $(1,12)$ or pain tolerance $(5,12)$ after stress manipulations. Others have also noted lack of effect of acute stress on pain threshold $(8,16)$. The diversity of effects that psychological stress has on pain perception may stem from variability in the pain induction methods $(24,35)$ and in the stress manipulation applied $(7,16)$. However, a more likely explanation for the diversity of reported stress effects on pain perception is the validity of the stress response. In very few studies was the stress response validated and corresponded with the revised, modern definition of stress (19), namely, an increase in perceived stress together with an increase in hypothalamic-pituitary-adrenal axis and/or sympathetic-adrenal-medullary variables. In the present study, the acute stress response was validated by both subjective and objective stress markers.

The mechanisms underlying SIH are not clear as yet. The connectivity between the stress system and the pain modulation systems can at least partly explain this effect. For example, the stimulation of the dorsomedial hypothalamic nucleus, known to mediate emotional stress, was shown to induce thermal hyperalgesia by recruiting the pain-facilitating "ON cells" in the rostral ventromedial medulla (22). There is also evidence that SIH is linked to secretion of cholecystokinin in the periaqueductal gray (21), which opposes, in turn, opioid-mediated analgesia (36). Similar mechanisms may be responsible for the reduction in pain threshold and in CPM among the triathletes after stress, especially because activity in the periaqueductal gray and in the medullary subnucleus reticularis dorsalis mediate such aspects of pain modulation $(39,40)$. In addition to the connectivity between the hypothalamus and the brain stem, the ventrolateral prefrontal cortex also seems crucial in implementing stress modulation of pain (38). Anxiety, for example, is accompanied by impaired ventrolateral prefrontal cortex functioning, leading to selective attention to threat, a biased interpretation of the situation and, consequently, pain aggravation (38). The $\mathrm{SIH}$ observed here may thus also stem from a variety of topdown influences on the link between brain structures involved in pain modulation and emotion modulation (for a review, see (17).

Low- versus high-stress responders. Interestingly, not all the triathletes responded to stress in a similar manner. When the triathletes were divided according to the magnitude of their stress response, low-stress responders had stronger $\mathrm{SIH}$ than high-stress responders. This was manifested as a decrease in pain tolerance among the former (that did not occur in high-stress responders) and a greater reduction in CPM (almost three fold that of high-stress responders). To understand these seemingly paradoxical results, we searched for additional differences between low- and high-stress responders. Such were observed in the baseline profile of the subjects. First, lowstress responders had better CPM and no TSP compared with high-stress responders, namely, they seemed to have a better pain inhibition profile at baseline. Second, low-stress responders had lower levels of perceived stress and anxiety at baseline and they also reported lower levels of perceived stress during training and competitions. In other words, they exhibited less negative emotions at baseline and in everyday life. In addition, the level of cortisol after the stress manipulation among low-stress responders was significantly lower compared with high-stress responders. 
Thus, the stronger SIH exhibited by low-stress responders could be a result of several factors. It is possible that people with more efficient pain modulation at baseline are affected the most by SIH simply because they have high ceiling levels that can only be reduced if affected. For example, if the stress manipulation is able to reduce CPM to a floor value of two VAS units, those who started the experiment with the CPM of approximately four VAS units will present a greater reduction in CPM than those who started the experiment with the CPM of only 2.5 VAS units. Although this explanation may be relevant to CPM, it cannot explain the between-group differences in pain tolerance because both subgroups started with the same tolerance level.

Alternatively but not mutually exclusive is the possibility that the level of the stress-induced arousal determined the level of SIH. Studies have suggested that SIH occurs under conditions of low to moderate arousal, whereas the opposite phenomenon SIA occurs under high arousal (for a review, see (27)). The theory pertains that increased arousal induces apprehensive anticipation of threat, which in turn induces pain inhibition because of increased defensive activation or directed attention to the more salient stimulus/threat (e.g., (30)). In line with this view, triathletes with lower arousal (lower-stressinduced levels of cortisol and negative emotion) had stronger $\mathrm{SIH}$ than triathletes with higher arousal. Low-stress responders were also less stressed out than high-stress responders at baseline/everyday life. Therefore, the acute stress manipulation might had a stronger effect on the former group because of the overall inherent reduced stress/arousal level in these subjects.

Weaker SIH among high-stress responders may also be related to the antinociceptive effects of cortisol. In the present study, the high-stress responders who exhibited increased cortisol levels had significantly less SIH than the low-stress responders in whom cortisol levels did not increase after the manipulation. Studies in animals show that high levels of corticosteroids reduce spinal excitability (29) and adrenalectomy increases the severity of hyperalgesia (13). In subjects, the elevation of corticosteroids, including cortisol reduce postoperative pain as well as chronic and acute pain ratings $(2,3,23)$. It is thus possible that the high cortisol level among high-stress responders counteracted the $\mathrm{SIH}$ because of its antinociceptive effects.

Triathletes versus nonathletes. Although both the triathletes in this study and nonathletes in our previous study (15) experienced SIH, it manifested itself somewhat differently. These groups are comparable because both triathletes and nonathletes were subject to the same stress manipulation and the same experimental protocol and conditions. Thus,

\section{REFERENCES}

1. al'Absi M, Nakajima M, Grabowski J. Stress response dysregulation and stress-induced analgesia in nicotine dependent men and women. Biol Psychol. 2013;93:1-8.

2. al'Absi M, Petersen KL, Wittmers LE. Adrenocortical and hemodynamic predictors of pain perception in men and women. Pain. 2002;96:197-204. only triathletes lowered their pain threshold and only nonathletes increased their TSP after the stress manipulation. In addition, triathletes exhibited a far greater reduction in CPM than nonathletes did. The CPM of triathletes in the present study reduced from 3.15 at baseline to 1.72 after stress, and the CPM of nonathletes in our previous study (15) reduced from 2.42 to 1.77 (delta of 1.43 vs 0.65 VAS units, respectively). These values show that triathletes have more efficient pain inhibition than nonathletes at baseline, also found with other athletes (e.g., $(28,35)$; however, see (34)) and highly physically active people $(25,37)$. Yet it appears that triathletes lose their advantageous CPM over nonathletes under psychological stress as CPM magnitude becomes similar to the CPM of nonathletes.

Here again, perhaps the initial ceiling levels of CPM among triathletes allow for a greater reduction in CPM after stress than that in nonathletes. Alternatively, perhaps the reactivity to stress of athletes in general, or toward the stressmanipulation used here (MIST) in particular, underlies the different SIH between triathletes and nonathletes. Triathletes are accustomed to physical and mental challenges and are highly motivated $(6,31)$. No doubt that the arithmetic tasks of the MIST and the constant time constraint present a challenge to both athletes and nonathletes. However, because the MIST manipulation involves an element of competition, triathletes may have underwent different emotional processing when required to "achieve the target" than nonathletes did.

In summary, this study shows that despite very efficient baseline pain modulation, triathletes respond to acute psychological stress by a significant SIH. Paradoxically, however, this SIH is more pronounced among triathletes with lower stress-reactivity perhaps because of initial ceiling levels of pain modulation or lower levels of arousal and cortisol elevation. Future studies might explore whether this reactivity pattern is universal to all athletes or depends on the type and intensity of sport. Despite the SIH after the MIST, triathletes do persevere in extreme efforts even if these involve considerable pain and stress. Whether it is a unique physical and/or psychological profile that enable this perseverance despite the involved stress is yet to be determined.

This work was performed in partial fulfillment of the requirements for a Ph.D. degree by Nirit Geva at the Sackler Faculty of Medicine, Tel Aviv University, Israel.

The authors declare no conflicts of interests and that the results of the present study do not constitute endorsement by the American College of Sports Medicine.

The authors declare that the results of the study are presented clearly, honestly, and without fabrication, falsification, or inappropriate data manipulation.

3. Allen LB, Lu Q, Tsao JC, Worthman CM, Zeltzer LK. Sex differences in the association between cortisol concentrations and laboratory pain responses in healthy children. Gender Med. 2009;6: 193-207.

4. Atkinson M. Triathlon, suffering and exciting significance. Leisure Studies. 2008;27:165-80. 
5. Bandura A, Cioffi D, Taylor CB, Brouillard ME. Perceived selfefficacy in coping with cognitive stressors and opioid activation. J Pers Soc Psychol. 1988;55:479-88.

6. Breiger J, Cumming SP, Smith RE, Smoll F, Brewer B. Winning, motivational climate, and young athletes' competitive experiences: some notable sex differences. Int J Sports Sci Coach. 2015;10:395-412.

7. Caceres C, Burns JW. Cardiovascular reactivity to psychological stress may enhance subsequent pain sensitivity. Pain. 1997;69:237-44.

8. Cathcart S, Winefield AH, Lushington K, Rolan P. Noxious inhibition of temporal summation is impaired in chronic tension-type headache. Headache. 2010;50:403-12

9. Crettaz B, Marziniak M, Willeke P, et al. Stress-induced allodynia - evidence of increased pain sensitivity in healthy humans and patients with chronic pain after experimentally induced psychosocial stress. PLoS One. 2013;8:e69460.

10. Dedovic K, Renwick R, Mahani NK, Engert V, Lupien SJ, Pruessner JC. The Montreal Imaging Stress Task: using functional imaging to investigate the effects of perceiving and processing psychosocial stress in the human brain. $J$ Psychiatry Neurosci. 2005;30:319-25.

11. Dickerson SS, Kemeny ME. Acute stressors and cortisol responses: a theoretical integration and synthesis of laboratory research. Psychol Bull. 2004;130:355-91.

12. Diener SJ, Wessa M, Ridder S, et al. Enhanced stress analgesia to a cognitively demanding task in patients with posttraumatic stress disorder. J Affective Dis. 2012;136:1247-51.

13. Fukuda T, Nishimoto C, Miyabe M, Toyooka H. Unilateral adrenalectomy attenuates hemorrhagic shock-induced analgesia in rats. J Anesthesia. 2007;21:348-53.

14. Geva N, Defrin R. Enhanced pain modulation among triathletes: a possible explanation for their exceptional capabilities. Pain. 2013; 154:2317-23.

15. Geva N, Pruessner J, Defrin R. Acute psychosocial stress reduces pain modulation capabilities in healthy men. Pain. 2014;155:2418-25.

16. Girdler SS, Maixner W, Naftel HA, Stewart PW, Moretz RL, Light KC. Cigarette smoking, stress-induced analgesia and pain perception in men and women. Pain. 2005;114:372-85.

17. Jennings EM, Okine BN, Roche M, Finn DP. Stress-induced hyperalgesia. Prog Neurobiol. 2014;121:1-18.

18. Koltyn KF. Analgesia following exercise. Sports Med. 2000;29(2):85-98.

19. Koolhaas JM, Bartolomucci A, Buwalda B, et al. Stress revisited: a critical evaluation of the stress concept. Neurosci Biobehav Rev. 2011;35(5):1291-1301.

20. Levine A, Zagoory-Sharon O, Feldman R, Lewis JG, Weller A. Measuring cortisol in human psychobiological studies. Physiol Behav. 2007;90:43-53.

21. Lovick TA. Pro-nociceptive action of cholecystokinin in the periaqueductal grey: a role in neuropathic and anxiety-induced hyperalgesic states. Neurosci Biobehav Rev. 2008;32:852-62.

22. Martenson ME, Cetas JC, Heinricher MM. A possible neural basis for stress induced hyperalgesia. Pain. 2009;142:236-44.

23. McEwen BS, Kalia M. The role of corticosteroids and stress in chronic pain conditions. Metabolism. 2010;59:S9-15.
24. Mogil JS, Bailey AL. Sex and gender differences in pain and analgesia. Prog Brain Res. 2010;186:141-57.

25. Naugle KM, Riley JL3rd. Self-reported physical activity predicts pain inhibitory and facilitatory function. Med Sci Sports Exercise. 2014;46(3):622-9.

26. Nilsen KB, Christiansen SE, Holmen LB, Sand T. The effect of a mental stressor on conditioned pain modulation in healthy subjects. Scand J Pain. 2012;3:142-8.

27. Olango WM, Finn DP. Neurobiology of stress-induced hyperalgesia. In: Taylor BK, Finn DP, editors. Behavioral Neurobiology of Chronic Pain. Berlin Heidelberg: Springer; 2014. pp. 251-80.

28. Ord P, Gijsbers K. Pain thresholds and tolerances of competitive rowers and their use of spontaneous self-generated pain-coping strategies. Percept Mot Skills. 2003;97:1219-22.

29. Pinto-Ribeiro F, Moreira V, Pêgo JM, Leão P, Almeida A, Sousa N. Antinociception induced by chronic glucocorticoid treatment is correlated to local modulation of spinal neurotransmitter content. Mol Pain. 2009;5:41.

30. Rhudy JL, Meagher MW. Fear and anxiety: divergent effects on human pain thresholds. Pain. 2000;84:65-75.

31. Smith AL, Ntoumanis N. An examination of goal motives and athletes' self-regulatory responses to unattainable goals. Int J Sport Psychol. 2014;45:538-58.

32. Spielberger CD, Gorsuch RL, Lushene R, Vagg PR, Jacobs GA. Manual for the State-Trait Anxiety Inventory (STAI) (Self Evaluation Questionnaire). Palo Alto (CA): Consulting Psychologists Press; 1983.

33. Staud R, Weyl EE, Riley JL 3rd, Fillingim RB. Slow temporal summation of pain for assessment of central pain sensitivity and clinical pain of fibromyalgia patients. PLoS One. 2014;9(2):e89086.

34. Tesarz J, Gerhardt A, Schommer K, Treede RD, Eich W. Alterations in endogenous pain modulation in endurance athletes: an experimental study using quantitative sensory testing and the coldpressor task. Pain. 2013;154:1022-9.

35. Tesarz J, Schuster AK, Hartmann M, Gerhardt A, Eich W. Pain perception in athletes compared to normally active controls: a systematic review with meta-analysis. Pain. 2012;153:1253-62.

36. Tortorici V, Nogueira L, Salas R, Vanegas H. Involvement of local cholecystokinin in the tolerance induced by morphine microinjections into the periaqueductal gray of rats. Pain. 2003;102:9-16.

37. Umeda M, Lee W, Marino CA, Hilliard SC. Influence of moderate intensity physical activity levels and gender on conditioned pain modulation. J Sports Sci. 2016;34:467-76.

38. Wiech K, Kalisch R, Weiskopf N, Pleger B, Stephan KE, Dolan RJ. Anterolateral prefrontal cortex mediates the analgesic effect of expected and perceived control over pain. J Neurosci. 2006; 26:11501-9.

39. Yarnitsky D. Conditioned pain modulation (the diffuse noxious inhibitory control-like effect): its relevance for acute and chronic pain states. Curr Opin Anesthesiol. 2010;23:611-5.

40. Youssef AM, Macefield VG, Henderson LA. Pain inhibits pain; human brainstem mechanisms. Neuroimage. 2016;124:54-62. 